\title{
RECOGNITION OF FACIAL EXPRESSIONS IN ADHD
}

The identification of facial expressions of four emotions (joy, anger, disgust, and sadness) was investigated in 30 children aged 7-12 years with ADHD, predominantly hyperactive-impulsive subtype, at Free University of Brussels, Belgium. Compared to 30 matched unimpaired control children, children with ADHD showed a general deficit in decoding emotional facial expressions depicted in 16 validated photographs, with a specific deficit in identifying anger and sadness. Patients lacked awareness of decoding errors as compared to control subjects. Interpersonal problems within the ADHD group were significantly correlated with impairment of emotional facial expression decoding. This nonverbal decoding disability in ADHD children has therapeutic implications, including a need for systematic verbal reinforcement. (Pele K, Kornreich C, Foisy M-L, Dan B. Recognition of emotional facial expressions in attention-deficit hyperactivity disorder. Pediatr Neuroi Aug 2006;35:93-97). (Respond: Dr Pele, Department of Neurology, Hopital Universitaire des Enfants Reine Fabiola, Free University of Brussels (ULB), 15 Avenue JJ Crocq, 1020 Brussels, Belgium).

COMMENT. Children with hyperactive-impulsive ADHD have an impaired ability to recognize emotional facial expressions, especially anger and sadness. This deficit in nonverbal language may be linked to visual-perceptual impairments, common in ADHD, and sometimes explained by dysfunction or damage to the nondominant parietal lobe and other cortical regions, including the temporal lobe and basal ganglia. The findings in the following article might indicate that the nondominant amygdala could be implicated in ADHD patients with impaired emotional facial expression.

\section{Facial emotion recognition after nondominant temporal lobectomy for epilepsy.}

Patients with temporal lobe epilepsy (TLE) associated with mesial temporal sclerosis (MTS) often incur damage to the right (nondominant) amygdala. Patients with nondominant anteromedial temporal lobectomy were divided into groups of early onset and late-onset TLE. They were tested by a standardized human face series compared to target faces expressing fear, anger, and happiness. Early nondominant amygdala insult, with TLE onset before age 6 years, results in impaired facial emotion recognition (FER), especially for frightened expression or fear. FER for anger and happiness are unaffected. Late onset TLE patients, after age 5 years, have no deficit in facial emotion recognition. Despite seizure control after temporal lobectomy, early onset TLE patients have persistent impairment of FER for frightened expression, whereas late TLE onset patients show no deficits in FER. Normal development of the right amygdala is required for optimal fear recognition. (McClelland S III, Garcia RE, Peraza DM et al. Epilepsia Aug 2006;47:1337-1342).

\section{GENETIC BASIS FOR ADHD SUBTYPES}

Parental questionnaires regarding symptoms of ADHD in 1480 twin pairs were mailed when children were 8-9, 13-14, and 16-17 years old. Symptoms of hyperactivityimpusivity declined with increasing age, whereas inattention was not age dependent. Persistent genetic variance was present primarily across subtypes of ADHD. (Larsson $\mathrm{H}$ et al. J Am Acad Child Adolesc Psychiatry August 2006;45:973-981). 